\title{
Mucin degrader Akkermansia muciniphila accelerates intestinal epithelial regeneration and repairs damaged intestinal mucosa
}

Mi-Na Kweon ( $\square$ mnkweon@amc.seoul.kr)

Asan Medical Center https://orcid.org/0000-0003-2916-2524

Seungil Kim

Asan Medical Center

Tae-Young Kim

Asan Medical Center

Yeji Kim

Asan Medical Center

Yong-Soo Lee

Asan Medical Center

Su-Hyun Lee

Asan Medical Center

Mi-Na Kim

Asan Medical Center

\section{Research}

Keywords: Gut microbiota, Akkermansia muciniphila, Intestinal stem cells, Lgr5, Mucin- degrading bacteria, G protein-coupled receptors, epithelial development, small intestine, organoids

Posted Date: May 20th, 2020

DOI: https://doi.org/10.21203/rs.3.rs-29112/v1

License: (a) (1) This work is licensed under a Creative Commons Attribution 4.0 International License.

Read Full License 


\section{Microbiome}

\section{Mucin degrader Akkermansia muciniphila accelerates intestinal epithelial regeneration and repairs damaged intestinal mucosa}

Seungil Kim ${ }^{1}$, Tae-Young Kim ${ }^{1}$, Yeji Kim ${ }^{1}$, Yong-Soo Lee ${ }^{1}$, Su-Hyun Lee ${ }^{1}$, Mi-Na Kim ${ }^{2}$, and Mi-Na Kweon ${ }^{1 *}$

${ }^{1}$ Mucosal Immunology Laboratory, Department of Convergence Medicine, ${ }^{2}$ Department of Laboratory Medicine, University of Ulsan College of Medicine / Asan Medical Center, Seoul 05505, Korea

Correspondence to

Prof. Mi-Na Kweon, Deparment of Convergence Medicine, Asan Medical Center, Seoul, Korea; mnkweon@amc.seoul.kr 


\section{Abstract}

Background: Mucin-degrading bacteria are densely populated in the intestinal epithelium; however, their interaction with intestinal stem cells (ISCs) and their progeny has not been elucidated. To determine whether mucin-degrading bacteria play a role in gut homeostasis, mice were treated with Akkermansia muciniphila, a specialized species that degrades mucin. A total of 32 fecal samples were obtained from healthy volunteers and A. muciniphila was isolated from 11 samples. Mechanism of A. muciniphila was observed in vivo and in vitro, and studied using organoids, histology, metagenomics, and whole genome sequencing.

Results: We found that administration of A. muciniphila for 4 weeks accelerated the proliferation of $\mathrm{Lgr5}^{+}$ISCs and promoted the differentiation of Paneth cells and goblet cells in the small intestine (SI). The levels of acetic and propionic acids were higher in the cecal contents of A. muciniphila-treated mice than in PBS-treated mice. SI organoids treated with cecal content supernatant obtained from A. muciniphila-treated mice were larger and could be diminished by treatment with $\mathrm{G}$ protein-coupled receptor $(\mathrm{Gpr}) 41 / 43$ antagonists. Pretreatment of mice with A. muciniphila reduced gut damage caused by radiation and methotrexate. A novel isotype of A. muciniphila strain was isolated from heathy human feces that possessed improved functions for intestinal epithelial regeneration.

Conclusions: These findings suggest that mucin-degrading bacteria (such as A. muciniphila) may play a crucial role in promoting ISC-mediated epithelial development and contribute to intestinal homeostasis maintenance.

Keywords: Gut microbiota, Akkermansia muciniphila, Intestinal stem cells, Lgr5, Mucindegrading bacteria, $\mathrm{G}$ protein-coupled receptors, epithelial development, small intestine, organoids 


\section{Background}

Mammalian intestinal epithelial cells (IECs) have a rapid turnover rate and are replenished every 3-5 days $[1,2]$. All types of IECs derive from intestinal stem cells (ISCs) that can generate either ISC daughters or proliferating progenitors called transit-amplifying (TA) cells $[3,4]$. TA cells terminally differentiate into secretory cell lineages, such as Paneth, goblet, enteroendocrine, and enterocytes [2]. Importantly, Paneth cells migrate down into the intestinal crypt bottom to produce such ISC niche factors as Wnt3, EGF, TGF $\alpha$, and Dll4 [5].

The Wnt signaling pathway plays an important role in promoting and driving the proliferative activity of ISCs and IEC differentiation [6, 7]. A recent study revealed that Wnt/ $\beta$-catenin signaling supports gut homeostasis by maintaining self-renewal of Lgr5-expressing stem cells in the intestinal crypts [8]. The Wnt ligand binds to the Frizzled and low-density lipoprotein receptor-related protein receptor. This leads to accumulation of $\beta$-catenin, the main mediator of the Wnt signal cascade in the gut [9], which translocates from the cytoplasm into the nucleus. Inside the nucleus, $\beta$-catenin binds with the transcription factor TCF to regulate genes involved in proliferation $[10,11]$.

Gut microbiota maintain gut homeostasis [12, 13]. Several studies have identified a link between dysbiosis and disease, such as inflammatory disorder, metabolic syndrome, and mental illness [14-16]. Metabolites produced by gut microbiota are proposed to modulate host physiology $[14,17,18]$. Short chain fatty acids (SCFAs, e.g., acetic, propionic, and butyric acids) are functional metabolites produced by bacterial fermentation of undigested complex carbohydrates [19]. By binding to G protein-coupled receptors (Gpr) 41 and 43, SCFAs can affect host gut immunity and metabolism [20, 21]. In addition, we recently 
suggested that gut microbiota-derived lactate promotes IEC development in a Gpr81dependent manner [22].

Akkermansia muciniphila is a mucin-degrading bacteria and the sole genus of phylum Verrucomicobia, which is found in human stools [23]. A. muciniphila represents approximately $1-3 \%$ of intestinal microbiota residing in the mucus layer near the IECs [24, 25]. Several studies have shown that the abundance of A. muciniphila is inversely correlated with various diseases, such as inflammatory bowel disease, diabetes, and obesity [26-28] Administration of A. muciniphila reduces weight gain and improves metabolic parameters such as glucose metabolism [27, 29-31]. Metformin, an anti-diabetic agent, increases the abundance of $A$. muciniphila in the gut microbiota of obese mice under diet conditions [32, 33]. A recent study also demonstrated a correlation between the clinical efficacy of immune checkpoint inhibitors and the relative abundance of A. muciniphila [34]. These results indicate that $A$. muciniphila may have potential as a key next-generation microbe with a wide spectrum of therapeutic applications.

In this study, we investigated the potential role for $A$. muciniphila in IEC development. Despite being a mucin-degrading bacterium, A. muciniphila paradoxically increased mucus production by promoting the differentiation of secretory IEC lineages. Administration of $A$. muciniphila enhanced ISC proliferation in a Gpr41/43-dependent manner and subsequently accelerated intestinal epithelial regeneration. Most importantly, A. mисіniphila protected mice from severe gut damage caused by radiation and chemotherapy. Taken together, our findings suggest that $A$. muciniphila promotes IEC development and maintains gut homeostasis. 


\section{Materials and methods}

\section{Mice}

The animals in this study were 8 - to- 10 -week old female mice that were fed sterile food and water ad libitum. C57BL/6 mice were purchased from OrientBio (Seong-Nam, South Korea) and Lgr5-EGFP-IRES-CreERT2 (Lgr5-GFP) mice were purchased from The Jackson Laboratory (Bar Harbor, ME). Mice were housed in the animal facility of the Asan Medical Center (Seoul, South Korea) and maintained under specific pathogen-free conditions. All animal experiments were approved by the Institutional Animal Care and Use Committee of Asan Medical Center (Approval No. 2019-12-251). All experiments were performed in accordance with relevant ethical guidelines and regulations.

\section{Isolation of $A$. muciniphila strains from human stool}

A total of 32 fecal samples were obtained from the human dock center of the Asan Medical Center. The samples were collected from fresh residual samples after fecal occult blood and parasitic examination on the same date (IRB No. A20201614). Fecal samples were suspended in PBS and then seeded onto brain heart infusion agar without dextrose (KisanBio) supplemented with $0.4 \%$ mucin (BHI-M). The fecal cultures were maintained at $37^{\circ} \mathrm{C}$ under anaerobic conditions generated using a GasPak 100 system (BD Bioscience). Approximately 50 colonies were selected from BHI-M plates and tested with a PCR for species-specific sequences with the primer set 5'-CAGCACGTGAAGGTGGGGAC-3' and 5'CCTTGCGGTTGGCTTCAGAT-3'. A. muciniphila was isolated from 11 samples. One strain 
was established per sample by sub-culturing for future experimentation.

\section{Oral administration of $A$. muciniphila}

A. muciniphila (ATCC BAA-835 ) and newly isolated A. muciniphila AK32 strains were cultured in brain heart infusion media (BD Bioscience) supplemented with $0.4 \%$ mucin (Sigma) and maintained in an anaerobic incubator using the GasPak 100 system (BD Bioscience) at $37^{\circ} \mathrm{C}$. Cultures were centrifuged, the culture pellet was suspended in anaerobic PBS, and the culture pellet was administered to mice $\left(8 \times 10^{8} \mathrm{CFU}\right.$ per dose $)$ every day for 4 weeks by oral Zonde needle.

\section{Treatment with irradiation and methotrexate}

Mice were injected intraperitoneally with methotrexate (MTX, $120 \mathrm{mg} / \mathrm{kg}$, Sigma) followed by administration of 10 Gy of total body irradiation (cesium source irradiator; Precision XRay, North Branford, CT).

\section{Cell isolation}

Mouse SIs were opened longitudinally and washed with PBS. To dissociate the crypts, tissues were incubated at $4^{\circ} \mathrm{C}$ in $1 \mathrm{mM}$ EDTA in PBS for $30 \mathrm{~min}$, washed in PBS, and then transferred into $5 \mathrm{mM}$ EDTA in PBS for an additional $1 \mathrm{hr}$ of incubation at $4^{\circ} \mathrm{C}$. Samples were then suspended in PBS and filtered by a 70- $\mu \mathrm{m}$ cell strainer (BD Falcon). To purify 
ISCs and Paneth cells from Lgr5-GFP mice, crypt cell suspensions were dissociated using TrypLE Express (Thermo Fisher Scientific) for $10 \mathrm{~min}$ at $37^{\circ} \mathrm{C}$. The dissociated cells were stained with the Live/Dead Cell Stain kit (Thermo Fisher Scientific) and anti-CD24 monoclonal antibody (Thermo Fisher Scientific). Cell sorting was performed using a FACS AriaIll cell sorter. ISCs were sorted as Lgr5-GFP ${ }^{\text {hi }}$ and Paneth cells were sorted as Lgr5$\mathrm{GFP}^{-} \mathrm{CD} 24^{\mathrm{hi}}$, respectively.

\section{Organoid culture}

For construction of organoids, 200-500 crypts per well were suspended in Matrigel (Corning) as described [35]. Complete ENR medium (all components from Thermo Fisher Scientific unless noted) comprised of advanced DMEM/F12 (Gibco), antibiotic-antimycotic $(\times 100), 1$ mM N-acetyl cysteine (Sigma-Aldrich), B27 supplement, N2 supplement, EGF, Noggin (R\&D Systems), R-spondin-1-conditioned medium, and Y-27632 (Sigma). Y-27632 were added to the ENR medium for the first 48-72 hr of culture only and then removed during the medium change. The ENR medium was replaced every 2-3 days. Isolated ISCs and Paneth cells were co-cultured in ENR medium supplemented with Jagged-1 (1 $\mu \mathrm{M}$, Anaspec). WntC59 (50 $\mu \mathrm{M}$, Abcam) was used as a porcupine (PORCN) inhibitor. The surface areas of SI organoids were measured microscopically by taking several random non-overlapping photos of organoids in a well using an inverted microscope (Carl Zeiss). Each photo was analyzed using ImageJ software (NIH) and the Zen image program (Carl Zeiss). Organoid perimeters for area measurements were defined manually using automated ImageJ software. 


\section{Histology}

Ileum tissues were removed, opened longitudinally, and formed into Swiss rolls. The tissue was then fixed in $4 \%$ paraformaldehyde (PFA) and embedded in paraffin. Tissue sections were stained with hematoxylin-eosin (H\&E) or periodic acid-Schiff (PAS).

\section{Immunofluorescence staining}

Ileum tissues were fixed with $4 \%$ PFA and dehydrated with $15 \%$ then $30 \%$ sucrose in PBS.

Dehydrated tissues were formed into a Swiss roll, frozen, and sliced. For staining, collected organoids were permeabilized in PBS containing $0.1 \%$ Tween 20 and blocked with $0.5 \%$ BSA in PBS for $1 \mathrm{hr}$. For Muc2 staining, ileum tissues containing feces were fixed in Carnoy's solution and embedded in paraffin. The primary antibodies used were rabbit antiMuc2 (Abcam), rat anti-Ki67 (Biolegend), rabbit anti-lysozyme (Abcam), goat anti-Wnt3 (Abcam), mouse anti- $\beta$-catenin (BD Bioscience), and mouse anti-phospho-ERK1/2 (Thermo Fisher Scientific). Secondary antibodies included Alexa Flour goat 594 anti-rat IgG (Biolegend), Alexa Fluor 488 goat anti-mouse IgG (Abcam), Alexa Fluor 546 donkey antigoat IgG (Thermo Fisher Scientific), and Alexa Fluor 488 goat anti-rabbit IgG (Thermo Fisher Scientific).

\section{Organoid treatment with cecal contents}


Cecal contents $(100 \mathrm{mg}$ ) were diluted in $1 \mathrm{ml}$ of serum-free DMEM/F12 (Gibco) medium and vortexed for $1 \mathrm{hr}$. The contents were centrifuged at 4,000 rpm for $10 \mathrm{~min}$ and supernatants were passed through a $0.22-\mu \mathrm{m}$ syringe filter (Pall Corp.) before cultivation. To address the effectiveness of cecal contents in promoting organogenesis, we used ENR media supplemented with cecal supernatant diluted to $0.01 \%$ in advanced DMEM/F12. For inhibition of Gpr41/43, Gpr43 antagonist (GLPG0974, 0.1 $\mu \mathrm{M}$, Tocris) and Gpr41 antagonist ( $\beta$-hydroxybutyrate, $3 \mathrm{mM}$, Sigma) were used.

\section{Microbiome data analysis pipeline}

Total DNA was extracted from feces using QIAamp DNA stool mini kits (Qiagen) in accordance with the manufacturer's instructions. For bacterial PCR amplification, primers targeting $341 \mathrm{~F}$ and $805 \mathrm{R}$ were used. The amplified product was purified and sequenced by Chunlab (Seoul, South Korea) with an Illumina Miseq Sequencing system (Illumina). Processing raw reads started with quality check and filtering of low-quality $(<\mathrm{Q} 25)$ reads by Trimmnomatic ver. 0.32. After a quality control pass, paired-end sequence data were merged together using VSEARCH version 2.13.4 using default parameters. Non-specific amplicons that did not encode 16S rRNA were detected by nhmmer in the HMMER software package version 3.2.1. We used the EzBioCloud 16S rRNA database for taxonomic assignment by precise pairwise alignment [36]. After chimeric filtering, reads that were not identified to the species level (with <97\% similarity) in the EzBioCloud database were compiled. OTUs with single reads (singletons) were omitted from further analysis. The alpha diversity (Shannon index) and beta diversity for the sample difference were estimated. A taxonomic cladogram 
was generated using LEfSe with a threshold of 2 on the logarithmic LDA score [37]. A relationship based on a Pearson correlation between gut microbiota and SCFAs was visualized using Calypso software [38].

\section{Whole genome sequencing}

The integrity of gDNA was tested by running an agarose gel electrophoresis. gDNA was quantified using the Quant-IT PicoGreen (Invitrogen). The sequencing libraries were then prepared according to the manufacturer's instructions $(20 \mathrm{~kb}$ template preparation and the BluePippin $^{\mathrm{TM}}$ Size-Selection System) using the PacBio DNA template Prep Kit 1.0. The libraries were quantified using Quant-IT PicoGreen and qualified using the high-sensitivity DNA chip (Agilent Technologies). Subsequently, the libraries were sequenced using PacBio P6C4 chemistry in 8-well-SMART Cell v3 in PacBio RSII. The genome of the AK32 strain was constructed de novo using PacBio sequencing data. Sequencing analysis was performed by Chunlab. PacBio sequencing data were assembled with PacBio SMRT analysis 2.3.0 using the HGAP2 protocol (Pacific Biosciences). Resulting contigs from PacBio sequencing data were circularized using Circlator 1.4.0 (Sanger Institute). The gene-finding and functional annotation pipeline of whole genome assembly was used from the EzbioCloud genome database. Protein coding sequences were predicted by Prodigal 2.6.2. Genes coding for tRNA were searched using tRNAscan-SE 1.3.1. The rRNA and other non-coding RNAs were searched by Rfam 12.0 database. Comparative whole genome analysis was studied by average nucleotide identity base BLAST (ANIb). The ANIb value was calculated by ANI calculator from Kostas lab (http://enve-omics.ce.gatech.edu/ani). Operons including $p d h$ and 
mmd were predicted by using MicrobesOnline Operon Predictions tools [39]. We estimated the -10 and -35 regions in promoter sequences of these genes by use of the phiSITE database [40].

\section{Real-time PCR}

Total RNA from the SI and SI-derived organoids was extracted using the RNeasy mini kit (Qiagen) and cDNA was synthesized using Superscript II reverse transcriptase and oligo dT primer (Thermo Fisher Scientific). Total RNA of A. muciniphila was extracted using Trizol (Thermo Fisher Scientific). The ReverTra Ace qPCR RT master mix with gDNA remover (Toyobo) was used to synthesis cDNA from bacterial RNA. cDNA was used as the template for real-time PCR performed using SYBR green chemistry (Thermo Fisher Scientific) on a Real Time PCR system (Applied Biosystems). The real-time PCR primers used in this study were as follows: Muc2, 5' - CCTTAGCCAAGGGCTCGGAA-3' and 5'GGCCCGAGAGTAGACCTTGG-3'; Lyz1, 5'- ATGGCGAACACAATGTCAAA-3' and 5'GCCCTGTTTCTGCTGAAGTC-3'; Dll1，5'- CAGGACCTTCTTTCGCGTAT-3' and 5'AAGGGGAATCGGATGGGGTT-3'; Mathl, 5'- GCCTTGCCGGACTCGCTTCTC-3' and 5'- TCTGTGCCATCATCGCTGTTAGGG-3'; Spdef1, 5'- CCGGTTGCCTGCTACTGTTC3' and 5'- GCCCATTGCTCCTGATGCT-3'; Wnt3, 5'- CTTCTAATGGAGCCCCACCT-3' and 5'- GAGGCCAGAGATGTGTACTGC-3'; Axin2, 5'- AACCTATGCCCGTTTCCTCT-3' and 5'- GAGTGTAAAGACTTGGTCCA-3'; Ctnnb1, 5'- ATGGAGCCGGACAGAAAAGC3' and 5'- TGGGAGGTGTCAACATCTTC-3'; Lgr5, 5'- CCTGTCCAGGCTTTCAGAAG-3' and $\quad 5^{\prime}-\quad$ CTGTGGAGTCCATCAAAGCA-3'; $\quad \beta$-actin, $\quad 5^{\prime}-$ 
TGGAATCCTGTGGCATCCATGAAAC-3'

and

TAAAACGCAGCTCAGTAACAGTCCG-3'; $p d h$, 5'- AACCGATTATTGAAGCGGCA-3' and 5'- ATATTGGCGGCTTCGTGAAA-3'; mmd, 5'- GACCAAGAAGGAACGCCTCA-3' and 5'- GTTCCGTCACCTTGCATTCG-3'; Universal 16S rDNA, 5'ACTCCTACGGGAGGCAGCAG-3' and 5'- ATtACCGCGGCTGCTGG-3'; and $A$. muciniphila 16S rDNA, 5'- CAGCACGTGAAGGTGGGGAC-3' and 5'CCTTGCGGTTGGCTTCAGAT-3’.

\section{Quantitative measurement of SCFAs}

All reagents and solvents for metabolite analysis were purchased from Sigma. Freeze-dried cecal contents $(10 \mathrm{mg})$ were homogenized vigorously with $400 \mu \mathrm{l}$ of internal standard solution $\left[1 \mathrm{mM}\right.$ propionic acid $\left(\mathrm{C}_{3}\right)-\mathrm{d}_{6}$ and $100 \mu \mathrm{M}$ butyric acid $\left.\left(\mathrm{C}_{4}\right)-\mathrm{d}_{7}\right]$ in water. For analysis of bacterial culture supernatant, $100 \mu \mathrm{L}$ of culture supernatant was mixed with 200 $\mu \mathrm{L}$ of internal standard solution. After centrifuging, the supernatant was filtered out. AABDSH (20 $\mu$ l of $20 \mathrm{mM})$, TPP $(20 \mu \mathrm{l}$ of $20 \mathrm{mM})$, and DPDS $(20 \mu \mathrm{L})$ in dichloromethane were added to the filtrate. The solution was incubated for $10 \mathrm{~min}$ at RT with vortexing and dried under vacuum. The sample was reconstituted with $80 \mu \mathrm{L}$ of methanol prior to LC-MS/MS analysis. The LC-MS/MS system was equipped with a 1290 HPLC (Agilent Technologies, Denmark), Qtrap 5500 (ABSciex), and a reverse-phase column (Pursuit 5 C18 $150 \times 2.0$ mm, Agilent Technologies). The extracted ion chromatogram (EIC) corresponding to a specific transition for each metabolite was used for quantitation. The area under the curve of each EIC was normalized to the EIC of the internal standard. The peak area ratio of each metabolite 
was normalized to the internal standard using serum volume or tissue weight in a sample and then used for relative comparison.

\section{Statistics}

Statistical analyses were performed with Prism software (GraphPad, La Jolla, CA). For pairwise and two independent group comparisons, a two-tailed t-test was used. Data are presented as mean \pm SEM. The values $p<0.05, p<0.01$, and $p<0.001$ were considered as statistically significant. 


\section{Results}

Oral administration of $A$. muciniphila may promote epithelial differentiation in the small intestine.

To address whether mucin-degrading bacteria regulate IEC differentiation, A. muciniphila was given orally to mice for 4 weeks. Colonization of A. muciniphila in the small intestine (SI) ileum was confirmed by in situ hybridization (ISH) (Figure S1A). Mice treated with A. muciniphila showed increased crypt height and higher numbers of mucin-producing goblet cells in the SI in comparison with mice treated with PBS (Figure 1A). Due to the increased presence of goblet cells in response to A. muciniphila treatment, Mucin 2 (Muc2) protein and mRNA expression was examined in the SI. Muc2 protein and mRNA expression were significantly higher in the SI of A. muciniphila-treated mice than in PBS-treated control mice (Figure 1B and 1C). Additionally, administration of A. muciniphila resulted in increased numbers of lysozyme-positive (Lyz $\left.{ }^{+}\right)$Paneth cells and mRNA expression of Lyzl in the crypt of the SI (Figure 1D and 1E). Previously, $\mathrm{Lgr}^{+} \mathrm{Ki}^{-} 7^{-}$cells located at crypt positions $+4 /+5$ in the SI were found to differentiate into secretory lineage cells [41]. In the current study, the percentage of $\mathrm{Lgr}^{+}{ }^{+} \mathrm{Ki} 67^{-}$cells located at the $+4 /+5$ crypt positions were significantly higher in A. muciniphila-treated mice than those in PBS-treated mice (Figure 1F and 1G). As predicted, we observed an increase in the expression of the transcription factors Dll1, Mathl, and Spdefl that regulate differentiation of secretory lineage cells [42-44] in A. muciniphilatreated mice compared with controls (Figure 1H). These results suggest that A. muciniphila may promote differentiation of secretory lineage cells in the SI. 
Oral administration of $A$. muciniphila may accelerate ISC proliferation.

Because secretory subtypes of IECs are derived from $\mathrm{Lgr}^{+}{ }^{+}$ISCs, we next investigated whether A. muciniphila modulates the proliferation of $\mathrm{Lgr5}^{+}$ISCs. Lgr5-GFP mice administered A. muciniphila had more GFP-expressing Lgr5 $^{+}$ISCs in the SI crypt than PBStreated mice (Figure 2A and 2B). Organoids derived from the SI crypt of A. muciniphilatreated mice were larger than those of PBS-treated mice (Figure 2C and 2D). Furthermore, Lgr5 expression was upregulated in the SI and SI organoids from A. muciniphila-treated mice compared with controls (Figure 2E). RNA ISH analysis indicated increased production of Olfm $4^{+}$ISCs, which were confirmed in the SI crypt of A. muciniphila-treated mice (Figure 2F). In addition, protein and mRNA levels of $M u c 2$ and $L y z 1$ were upregulated in the SI organoids of A. muciniphila-treated mice compared with PBS-treated mice (Figure 2G and $2 \mathrm{H})$. These results imply that A. muciniphila may play a critical role in accelerating the proliferation of ISCs.

Oral administration of $A$. muciniphila enhances ISC proliferation by Wnt signaling.

Because Wnt signaling is involved in maintaining ISC stemness in the SI crypt, we next investigated whether A. muciniphila treatment regulates the Wnt pathway. Mice given A. muciniphila orally had increased expression of Wnt3, Axin2, and Ctnnb1 in their SI tissues (Figure 3A). The upregulated expression of Wnt3 and Axin2 in the SI crypt of A. muciniphilatreated mice was further confirmed by RNA ISH (Figure 3B). In addition, Wnt3 protein levels were higher in the SI crypt and SI organoids of A. muciniphila-treated mice than in PBS-treated control mice (Figure 3C and S1B). Of note, $\beta$-catenin protein levels were upregulated in the nuclei of the SI crypt of A. muciniphila-treated mice compared with PBS- 
treated mice (Figure 3D) indicating increased translocation from the cytoplasm. Because the Wnt/ $\beta$-catenin pathway activates RAS-ERK signaling that in turn promotes stemness [45], we next examined ERK phosphorylation (pERK) in the SI crypt. As expected, oral administration with $A$. muciniphila increased pERK expression in the SI crypt compared with controls (Figure S1C). To address whether A. muciniphila activates Wnt3 signaling in the SI, Lgr5-GFP ${ }^{\text {hi }}$ ISCs isolated from naive mice were co-cultured with Paneth cells isolated from $A$. muciniphila-treated or PBS-treated mice. Interestingly, SI organoids grew significantly more in co-cultures isolated from A. muciniphila-treated mice than in PBS-treated mice (Figure 3E and 3F). This effect was diminished when the porcupine inhibitor (Wnt-C59) was added to the co-cultures (Figure 3E and 3F). Together, these results demonstrate that A. muciniphila promotes the secretion of Wnt3 from Paneth cells that support ISC proliferation in the SI.

Oral administration of $A$. muciniphila promotes SCFA secretion and ISC-mediated epithelial development.

Because microbiota-derived metabolites are a key factor in gut homeostasis, we next investigated whether metabolites produced from A. muciniphila treatment effected ISCmediated epithelial development. To address this question, the cecal contents from PBS- or $A$. muciniphila-treated mice were isolated and then applied to SI organoids from naive B6 mice. SI organoids treated with A. muciniphila-treated mouse cecum were significantly larger than those treated with PBS-treated cecum (Figure 4A). Furthermore, the mRNA levels of Lgr5, Lyz1, Muc2, and Wnt-related genes (Wnt3, Axin2, Ctnnb1) increased in the presence of cecal contents obtained from A. muciniphila-treated mice compared with PBS-treated mice (Figure 4B). To identify which metabolites are associated with A. muciniphila-mediated epithelial 
development, the levels of SCFAs were examined in cecal contents. As predicted, higher levels of SCFAs, including acetic, propionic, and butyric acids, were present in the cecal contents from A. muciniphila-treated mice than from PBS-treated mice (Figure 4C). Of these, acetic and propionic acids, but not butyric acid, were highly associated with increased SI organoid growth (Figure S2A and S2B). Treatment with the Gpr41/43 antagonist reduced SI organoid growth (Figure 4D), suggesting that A. muciniphila-derived SCFA metabolites play an important role in ISC-mediated epithelial development.

Oral administration of $A$. muciniphila alters gut microbiota composition and SCFA production.

We next addressed whether oral administration of $A$. muciniphila altered the composition of gut microbiota and, as predicted, found that it did (Figure S3A). At the phylum level, the gut microbiota from A. muciniphila-treated mice showed an increased proportion of phyla Bacteroidetes and Proteobacteria and decreased numbers of phyla Firmicutes, compared with PBS-treated mice (Figure S3B). Further, linear discriminant analysis (LDA) with LEfSe confirmed that several bacteria genera were prominently changed after A. muciniphila treatment (Figure 5A and 5B). The genera Muribaculum, Alistipes, Akkermansia, Helicobacter, and Desulfovibrio showed an upper 2 LDA score after A. muciniphila treatment compared with control mice (Figure 5B). Furthermore, the Shannon index was significantly increased in A. muciniphila-treated mice compared with PBS-treated mice, indicating alteration of the bacterial community structure (Figure 5C). Unifrac-based PCoA analysis demonstrated that the two groups were clustered separately (Figure 5D). Interestingly, a positive correlation was observed between the Akkermansia-induced population and presence 
of SCFA metabolites (acetic, propionic, and butyric acids) (Figure 5E). In summary, these results suggest that $A$. muciniphila treatment promotes ISC-mediated epithelial development by altering the gut microbiota composition that in turn activates SCFA secretion.

Oral administration of $A$. muciniphila may repairs radiation and chemotherapy gut damage.

Since A. muciniphila promotes ISC-mediated epithelial development, we next investigated whether A. muciniphila plays a role in preventing gut damage. Our previous study [22] showed that radiation (R; $10 \mathrm{~Gy})$ and methotrexate (M; MTX) cause severe damage to mouse SI tissues. In this study, we assessed PBS-treated mice $(\mathrm{PBS}+\mathrm{R}+\mathrm{M})($ Figure $6 \mathrm{~A}$ and $6 \mathrm{~B})$ and mice treated with A. muciniphila for 4 weeks prior to radiation and MTX treatment $(A$. muciniphila+R+M). The treated group had less severe damage (Figure 6B). In addition, more Lgr5 $^{+}$ISCs were maintained in the SI crypt of A. muciniphila+R+M mice compared with the $\mathrm{PBS}+\mathrm{R}+\mathrm{M}$ mice (Figure 6C). As predicted, A. muciniphila $+\mathrm{R}+\mathrm{M}$ mice lost less weight than the PBS+R+M mice (Figure 6D). The organoid size and number derived from the SI of $A$. muciniphila $+\mathrm{R}+\mathrm{M}$ mice were significantly increased in comparison with $\mathrm{PBS}+\mathrm{R}+\mathrm{M}-\mathrm{derived}$ SI organoids, indicating that pre-treatment with A. muciniphila reduced damage and may play a protective role in the gut (Figure $6 \mathrm{E}$ and $6 \mathrm{~F}$ ). These results suggest that the symbiotic actions of A. muciniphila may promote gut repair following damage provoked by cancer therapy.

A. muciniphila AK32 from healthy human feces is superior to BAA-835 for ISCmediated epithelial development. 
We next investigated whether an A. muciniphila strain isolated from healthy human feces promotes ISC-mediated epithelial development compared with the common strain (ATCC BAA- $835^{\mathrm{T}}$ ). By use of selective media and species-specific PCR analysis, we obtained 11 different A. muciniphila strains. To evaluate the effect of A. muciniphila on ISC-mediated epithelial development, SI-derived organoids were cultured with culture supernatant from one of the strains. Only treatment with the AK32 strain significantly increased organoid size (Figure 7A). To address whether increased ISC-mediated epithelial development by the AK32 strain is dependent on SCFAs, the Gpr41/43 antagonist was applied to the cultures. Treatment with the Gpr41/43 antagonist significantly reversed the AK32-mediated effect on SI-derived organoid size (Figure 7B). As anticipated, treatment with the AK32 increased production of acetic acids and propionic acids compared with the A. muciniphila type strain (Figure S4A). To examine how the AK32 strain increased SCFA secretion, the expression levels of two important enzymes, pyruvate dehydrogenase E1 component (Pdh) and $\mathrm{Na}^{+}$translocating methylmalonyl-CoA/oxaloacetate decarboxylase (Mmd) were examined (Figure S4C). The mRNA expression levels of $p d h$ and $m m d$ from the AK32 strain were higher than those of BAA-835 type strain (Figure 7C). Next, we used whole genome sequencing to analyze genetic characteristics of strain AK32, including the $p d h$ and $m m d$ coding genes. Figure S5 shows a complete genome map of AK32-based Clusters of Orthologous Groups (COG). The genomic characteristics (genome size and number of coding sequences) in the AK32 strain differed from those of type strain BAA-835 (Table S1). As the mRNA expression differences might be attributed to regulation of transcription by promoter sequences, we assessed the operons, including $p d h$ and $m m d$. Interestingly, strains AK32 and BAA-835 had the same promoter sequences and amino acid sequences of $p d h$ but those of $m m d$ differed (Figures S6 and S7). To further examine the in vivo function of strain AK32, mice were treated with either 
the AK32 strain or BAA-835 for 4 weeks. Of note, the SI organoids from AK32-treated mice were significantly larger those from mice treated with the BAA-835 type strain (Figure 7D). Administration of the AK32 strain increased SI crypt height and the number of mucinproducing goblet cells compared with the SI of mice treated with BAA-835 (Figure 7E). Administration of strain AK32 resulted in increased mRNA expression of Lgr5, Lyz1, Muc2, Wnt3, Axin2, and Ctnnb1 in the SI compared with findings in mice treated with strain BAA835 (Figure 7F). Furthermore, higher levels of acetic and propionic acids were detected in the cecal contents from AK32-treated mice than in mice treated with BAA-835 (Figure S4B). Thus, we concluded that the newly identified A. muciniphila AK32 strain was superior to A. muciniphila BAA-835 in terms of ISC-mediated epithelial development. 


\section{Discussion}

In this study, A. muciniphila was found to play a crucial role in ISC-mediated epithelial development by activation of the Wnt signaling pathway and repair of the damaged gut. Treatment with A. muciniphila upregulated the expression of genes involved in the Wnt signaling pathway and increased production of SCFA metabolites, such as acetic and propionic acids, which in turn maintain stemness of the ISCs. A novel A. muciniphila strain was isolated from healthy human stools that promoted the expression of genes involved in acetic acid and propionic acid production, and therefore may have improved functionality for maintaining gut homeostasis.

The $\operatorname{Lgr} 5^{+} \mathrm{Ki}^{-}$cells located at crypt positions $+4 /+5$ of the SI are destined to differentiate into secretory lineage cells [41]. Administration of A. muciniphila resulted in an increased density of $\mathrm{Lgr}^{+} \mathrm{Ki}^{-}$cells in the SI crypt, which led to an increased number of secretory lineage cells, such as goblet and Paneth cells. The most important pathway for IEC development is Wnt signaling $[6,46]$. In support of this theory, inhibition of the Wnt pathway was shown to reduce the number of $\mathrm{Math}^{+}$precursor cells, resulting in a depletion of secretory lineage cells [7]. Paneth cells play a critical role in maintaining the ISC niche and produce Wnt3 [5]. In this study, the co-culture of ISCs and Paneth cells demonstrated that Paneth cells from A. muciniphila-treated mice directly promoted ISC proliferation in a Wnt3dependent manner. Thus, we speculate that metabolites produced by A. muciniphila stimulate Paneth cells to secrete Wnt3, which then promotes the proliferation of $\mathrm{Lgr}^{+} \mathrm{Ki}^{-} 7^{-}$cells at SI crypt positions $+4 /+5$.

A previous study reported that $A$. muciniphila uses mucin as an energy source by 
converting it into acetic and propionic acids [47]. Paradoxically, others have found that $A$. muciniphila promotes the generation of mucin-secreting goblet cells that were depleted by a high-fat diet $[27,32,48]$. In this study, our aim was to investigate how A. muciniphila activates mucin secretion. We propose that acetic and propionic acids produced by $A$. muciniphila may be key factors for supporting the maturation of mucin-secreting goblet cells.

Another study reported that administration of A. muciniphila did not reconstitute the gut microbiome [31]. In contrast, we found that A. muciniphila altered the gut microbiota composition and structure, which may affect the pattern of metabolite secretion. Germ-free mice supplemented with A. muciniphila showed an exacerbated infection by Salmonella typhimurium, suggesting that an over-abundance of $A$. muciniphila and reduced microbiome diversity leads to a deleterious modification of the gut environment [49]. Also, an accumulation of antimicrobial peptide produced by Paneth cells may contribute to a change in gut microbiota [50]. We therefore hypothesize that treatment with A. muciniphila may alter the bacterial composition and SCFA production in the gut; for example, by altering the abundance of phyla Bacteroidetes, which produces acetic and propionic acids [51], or Alistipes and Rikenellaceae, which produce SCFAs [52, 53]. Therefore, we conclude that $A$. muciniphila increases SCFA production directly via mucin degradation and indirectly by altering the microbiome composition.

Individual bacterial strains, even the same species, have strain-specific abilities. Because lactic acid bacterial strains of the same species show different enzyme activity [54], we wondered if different A. muciniphila strains might have different effects on ISC-mediated epithelial development. We found that the newly identified A. muciniphila AK32 strain activated the expression of genes involved in SCFA production and increased the secretion of 
acetic and propionic acids. As expected, promoter sequences of mmd in AK32 differed by type strain, but those of $p d h$ were highly conserved. The detailed mechanism by which the expression occurs is yet to be determined. We did identify a strain-specific alteration of the Mmd amino acid sequence, which may influence the enzyme activity by changing propionic acid levels. Thus, we conclude that strain AK32 has a characteristic genome, unlike the $A$. muciniphila type strain, that leads to increased SCFA production, especially acetic and propionic acids.

Treatment with A. muciniphila had a greater effect on the production of propionic acid than on acetic acid. Previously, it was suggested that $A$. muciniphila and propionic acid regulate the expression of genes associated with the host lipid metabolism and activate the epigenome [55]. Accumulating evidence suggests that propionic acid may modulate the host physiology in several ways. For example, propionic acid stimulates the release of peptide YY and glucagon-like peptide-1 in human colonic cells, and thereby reduces energy intake and weight gain [56]. Intriguingly, propionic acid stimulates Muc2 production by IECs by regulating the expression of the prostaglandins [57]. A recent study proposed that supplementation of propionic acid improves the Treg/Th17 imbalance in multiple sclerosis patients [58]. Taken together with our results, we conclude that propionic acid may play an important role in IEC homeostasis and the overall gut and therefore may modulate host physiology.

Several studies have reported an interaction between gut metabolites and IEC development $[22,59]$. Our prior study revealed that microbiota-derived lactate promotes IEC development [22]. By contrast, no significant changes in lactate were found in mice treated with A. muciniphila, suggesting that lactate is not a crucial metabolite involved in $A$. 
muciniphila-mediated IEC development (Figure S8a). A previous study reported that fatty acids such as palmitic acid, the main metabolite produced by gut microbiota, enhanced ISC proliferation [59]. Furthermore, our recent study demonstrated that dietary cellulose prevented gut inflammation by increasing the A. muciniphila population and modulating production of lipid metabolites [60]. Taken together, A. muciniphila treatment may increase the production of lipid metabolites, including myristic and palmitic acid, which influence IEC development (Figure S8b). Further investigation is warranted to rule out this possibility. 


\section{Conclusions}

This study demonstrates that $A$. muciniphila likely plays a crucial role in IEC development and may be a potential clinical therapeutic for preventing gut damage. To our knowledge, this is the first study to show the direct effects of $A$. muciniphila on ISC-mediated epithelium development. A new A. muciniphila strain was identified from healthy human stools with improved homeostatic functionality, such as increased production of acetic and propionic acids. Furthermore, this study may be useful basis for identifying and evaluating human microorganisms with therapeutic potential. 


\section{Abbreviations}

ISC: Intestinal stem cell; IEC: Intestinal epithelial cell; Lgr5: Leucine-rich repeat-containing G-protein coupled receptor 5; SI: Small intestine; SCFA: Short chain fatty acid; Gpr: Gprotein coupled receptor; BHI: Brain heart infusion; Muc2: Mucin2; Lyz; Lysozyme; LEfSe: Linear discriminant analysis effect size; LDA: Linear discriminant analysis; MTX: Methotrexate

\section{Declarations}

\section{Ethics approval and consent to participate}

All animal experiments were approved by the Institutional Animal Care and Use Committee of Asan Medical Center (Approval No. 2019-12-251). Fecal samples were obtained from the human dock center of the Asan Medical Center under IRB approval No. A20201614 approved by Asan Medical Center Institutional Review Board.

\section{Consent for publication}

Not applicable.

\section{Availability of data and materials}


All microbiome data is publicly available in NCBI (BioProject ID PRJNA625127 and PRJNA632722).

\section{Competing interests}

The authors declare that they have no competing interests.

\section{Funding}

This work was supported by the National Research Foundation of Korea (NRF2019M3C9A6082487 and NRF-2020R1A2B5B03001450).

\section{Authors' contributions}

S. K. and M.-N. Kweon conceived the project, designed and performed experiments, and wrote the manuscript; T.-Y. K., Y. K., S.-H. L., Y.-S. L. performed experiments; M.-N. Kim. supported human experiments.

\section{Acknowledgements}

We thank the Metabolomics and FACS cores at the Convergence Medicine Research Center, Asan Medical Center, for their support and equipment. 


\section{References}

1. Barker N: Adult intestinal stem cells: critical drivers of epithelial homeostasis and regeneration. Nat Rev Mol Cell Biol2014, 15(1):19-33.

2. Clevers H: The intestinal crypt, a prototype stem cell compartment. Cel/2013, 154(2):274-284.

3. Bjerknes $\mathrm{M}$, Cheng $\mathrm{H}$ : Clonal analysis of mouse intestinal epithelial progenitors. Gastroenterology 1999, 116(1):7-14.

4. Barker N, van Es JH, Kuipers J, Kujala P, van den Born M, Cozijnsen M, Haegebarth A, Korving J, Begthel H, Peters PJ et al: Identification of stem cells in small intestine and colon by marker gene Lgr5. Nature 2007, 449(7165):1003-1007.

5. Sato T, van Es JH, Snippert HJ, Stange DE, Vries RG, van den Born M, Barker N, Shroyer $\mathrm{NF}$, van de Wetering $\mathrm{M}$, Clevers $\mathrm{H}$ : Paneth cells constitute the niche for Lgr5 stem cells in intestinal crypts. Nature 2011, 469(7330):415-418.

6. Korinek V, Barker N, Moerer P, van Donselaar E, Huls G, Peters PJ, Clevers H: Depletion of epithelial stem-cell compartments in the small intestine of mice lacking Tcf-4. Nat Genet 1998, 19(4):379-383.

7. Pinto D, Gregorieff A, Begthel H, Clevers H: Canonical Wnt signals are essential for homeostasis of the intestinal epithelium. Genes Dev 2003, 17(14):1709-1713.

8. Farin HF, Van Es JH, Clevers H: Redundant sources of Wnt regulate intestinal stem cells and promote formation of Paneth cells. Gastroenterology 2012, 143(6):1518-1529 e1517.

9. Ireland H, Kemp R, Houghton C, Howard L, Clarke AR, Sansom OJ, Winton DJ: Inducible Cre-mediated control of gene expression in the murine gastrointestinal tract: effect of loss of beta-catenin. Gastroenterology 2004, 126(5):1236-1246.

10. Behrens J, von Kries JP, Kuhl M, Bruhn L, Wedlich D, Grosschedl R, Birchmeier W: Functional interaction of beta-catenin with the transcription factor LEF-1. Nature 1996, 382(6592):638-642.

11. van de Wetering M, Sancho E, Verweij C, de Lau W, Oving I, Hurlstone A, van der Horn K, Batlle E, Coudreuse D, Haramis AP et at: The beta-catenin/TCF-4 complex imposes a crypt progenitor phenotype on colorectal cancer cells. Cel/2002, 111(2):241-250.

12. Belkaid Y, Hand TW: Role of the microbiota in immunity and inflammation. Cell 2014, 157(1):121-141.

13. Skelly AN, Sato Y, Kearney S, Honda K: Mining the microbiota for microbial and metabolitebased immunotherapies. Nat Rev Immuno/2019, 19(5):305-323.

14. Mazmanian SK, Round JL, Kasper DL: A microbial symbiosis factor prevents intestinal inflammatory disease. Nature 2008, 453(7195):620-625.

15. Turnbaugh PJ, Ley RE, Mahowald MA, Magrini V, Mardis ER, Gordon JI: An obesity- 
associated gut microbiome with increased capacity for energy harvest. Nature 2006, 444(7122):1027-1031.

16. Valles-Colomer M, Falony G, Darzi Y, Tigchelaar EF, Wang J, Tito RY, Schiweck C, Kurilshikov $\mathrm{A}$, Joossens $\mathrm{M}$, Wijmenga $\mathrm{C}$ et al: The neuroactive potential of the human gut microbiota in quality of life and depression. Nat Microbio/2019, 4(4):623-632.

17. Furusawa Y, Obata Y, Fukuda S, Endo TA, Nakato G, Takahashi D, Nakanishi Y, Uetake C, Kato $\mathrm{K}$, Kato $\mathrm{T}$ et al: Commensal microbe-derived butyrate induces the differentiation of colonic regulatory T cells. Nature 2013, 504(7480):446-450.

18. Koeth RA, Wang Z, Levison BS, Buffa JA, Org E, Sheehy BT, Britt EB, Fu X, Wu Y, Li L et al: Intestinal microbiota metabolism of L-carnitine, a nutrient in red meat, promotes atherosclerosis. Nat Med 2013, 19(5):576-585.

19. Miller TL, Wolin MJ: Pathways of acetate, propionate, and butyrate formation by the human fecal microbial flora. App/ Environ Microbio/ 1996, 62(5):1589-1592.

20. Macia L, Tan J, Vieira AT, Leach K, Stanley D, Luong S, Maruya M, lan McKenzie C, Hijikata A, Wong $C$ et al: Metabolite-sensing receptors GPR43 and GPR109A facilitate dietary fibreinduced gut homeostasis through regulation of the inflammasome. Nat Commun 2015, 6:6734.

21. Maslowski KM, Vieira AT, Ng A, Kranich J, Sierro F, Yu D, Schilter HC, Rolph MS, Mackay F, Artis $\mathrm{D}$ et al: Regulation of inflammatory responses by gut microbiota and chemoattractant receptor GPR43. Nature 2009, 461(7268):1282-1286.

22. Lee YS, Kim TY, Kim Y, Lee SH, Kim S, Kang SW, Yang JY, Baek IJ, Sung YH, Park YY et al. Microbiota-derived lactate accelerates intestinal stem-cell-mediated epithelial development. Cell Host Microbe 2018, 24(6):833-846 e836.

23. Cani PD, de Vos WM: Next-generation beneficial microbes: the case of Akkermansia muciniphila. Front Microbio/ 2017, 8:1765.

24. Derrien M, Vaughan EE, Plugge CM, de Vos WM: Akkermansia muciniphila gen. nov., sp. nov., a human intestinal mucin-degrading bacterium. Int J Syst Evol Microbiol 2004, 54(Pt 5):1469-1476.

25. Derrien M, Collado MC, Ben-Amor K, Salminen S, de Vos WM: The Mucin degrader Akkermansia muciniphila is an abundant resident of the human intestinal tract. App/ Environ Microbio/ 2008, 74(5):1646-1648.

26. Schneeberger M, Everard A, Gomez-Valades AG, Matamoros S, Ramirez S, Delzenne NM, Gomis R, Claret M, Cani PD: Akkermansia muciniphila inversely correlates with the onset of inflammation, altered adipose tissue metabolism and metabolic disorders during obesity in mice. Sci Rep 2015, 5:16643.

27. Everard A, Belzer C, Geurts L, Ouwerkerk JP, Druart C, Bindels LB, Guiot Y, Derrien M, Muccioli GG, Delzenne NM et at: Cross-talk between Akkermansia muciniphila and intestinal epithelium controls diet-induced obesity. Proc Natl Acad Sci U S A 2013, 110(22):9066-9071. 
28. Png CW, Linden SK, Gilshenan KS, Zoetendal EG, McSweeney CS, Sly LI, McGuckin MA, Florin TH: Mucolytic bacteria with increased prevalence in IBD mucosa augment in vitro utilization of mucin by other bacteria. Am J Gastroentero/2010, 105(11):2420-2428.

29. Chevalier C, Stojanovic O, Colin DJ, Suarez-Zamorano N, Tarallo V, Veyrat-Durebex C, Rigo D, Fabbiano S, Stevanovic A, Hagemann $S$ et at: Gut microbiota orchestrates energy homeostasis during cold. Cel/2015, 163(6):1360-1374.

30. Plovier H, Everard A, Druart C, Depommier C, Van Hul M, Geurts L, Chilloux J, Ottman N, Duparc $T$, Lichtenstein $L$ et al: A purified membrane protein from Akkermansia muciniphila or the pasteurized bacterium improves metabolism in obese and diabetic mice. Nat Med 2017, 23(1):107-113.

31. Depommier C, Everard A, Druart C, Plovier H, Van Hul M, Vieira-Silva S, Falony G, Raes J, Maiter D, Delzenne NM et at. Supplementation with Akkermansia muciniphila in overweight and obese human volunteers: a proof-of-concept exploratory study. Nat Med 2019, 25(7):1096-1103.

32. Shin NR, Lee JC, Lee HY, Kim MS, Whon TW, Lee MS, Bae JW: An increase in the Akkermansia spp. population induced by metformin treatment improves glucose homeostasis in diet-induced obese mice. Gut 2014, 63(5):727-735.

33. Lee H, Ko G: Effect of metformin on metabolic improvement and gut microbiota. App/ Environ Microbio/ 2014, 80(19):5935-5943.

34. Routy B, Le Chatelier E, Derosa L, Duong CPM, Alou MT, Daillere R, Fluckiger A, Messaoudene M, Rauber C, Roberti MP et at: Gut microbiome influences efficacy of PD-1based immunotherapy against epithelial tumors. Science 2018, 359(6371):91-97.

35. Sato T, Vries RG, Snippert HJ, van de Wetering M, Barker N, Stange DE, van Es JH, Abo A, Kujala P, Peters PJ et al: Single Lgr5 stem cells build crypt-villus structures in vitro without a mesenchymal niche. Nature 2009, 459(7244):262-265.

36. Yoon SH, Ha SM, Kwon S, Lim J, Kim Y, Seo H, Chun J: Introducing EzBioCloud: a taxonomically united database of 16S rRNA gene sequences and whole-genome assemblies. Int J Syst Evol Microbio/2017, 67(5):1613-1617.

37. Segata N, Izard J, Waldron L, Gevers D, Miropolsky L, Garrett WS, Huttenhower C: Metagenomic biomarker discovery and explanation. Genome Bio/2011, 12(6):R60.

38. Zakrzewski M, Proietti C, Ellis JJ, Hasan S, Brion MJ, Berger B, Krause L: Calypso: a userfriendly web-server for mining and visualizing microbiome-environment interactions. Bioinformatics 2017, 33(5):782-783.

39. Price MN, Huang KH, Alm EJ, Arkin AP: A novel method for accurate operon predictions in all sequenced prokaryotes. Nucleic Acids Res 2005, 33(3):880-892.

40. Klucar L, Stano M, Hajduk M: phiSITE: database of gene regulation in bacteriophages. Nucleic Acids Res 2010, 38(Database issue):D366-370. 
41. Basak O, van de Born M, Korving J, Beumer J, van der Elst S, van Es JH, Clevers H: Mapping early fate determination in Lgr5+ crypt stem cells using a novel Ki67-RFP allele. EMBO J 2014, 33(18):2057-2068.

42. van Es JH, Sato T, van de Wetering M, Lyubimova A, Yee Nee AN, Gregorieff A, Sasaki N, Zeinstra $\mathrm{L}$, van den Born $\mathrm{M}$, Korving $\mathrm{J}$ et at. Dll1+ secretory progenitor cells revert to stem cells upon crypt damage. Nat Cell Biol 2012, 14(10):1099-1104.

43. Yang Q, Bermingham NA, Finegold MJ, Zoghbi HY: Requirement of Math1 for secretory cell lineage commitment in the mouse intestine. Science 2001, 294(5549):2155-2158.

44. Gregorieff A, Stange DE, Kujala P, Begthel H, van den Born M, Korving J, Peters PJ, Clevers $\mathrm{H}$ : The ets-domain transcription factor Spdef promotes maturation of goblet and paneth cells in the intestinal epithelium. Gastroenterology 2009, 137(4):1333-1345 e1331-1333.

45. Wong VW, Stange DE, Page ME, Buczacki S, Wabik A, Itami S, van de Wetering M, Poulsom $\mathrm{R}$, Wright NA, Trotter MW et at. Lrig1 controls intestinal stem-cell homeostasis by negative regulation of ErbB signalling. Nat Cell Bio/ 2012, 14(4):401-408.

46. van der Flier LG, Clevers $\mathrm{H}$ : Stem cells, self-renewal, and differentiation in the intestinal epithelium. Annu Rev Physiol 2009, 71:241-260.

47. de Vos WM: Microbe Profile: Akkermansia muciniphila: a conserved intestinal symbiont that acts as the gatekeeper of our mucosa. Microbiology 2017, 163(5):646-648.

48. Cani PD, Everard A, Duparc T: Gut microbiota, enteroendocrine functions and metabolism. Curr Opin Pharmaco/2013, 13(6):935-940.

49. Ganesh BP, Klopfleisch R, Loh G, Blaut M: Commensal Akkermansia muciniphila exacerbates gut inflammation in Salmonella Typhimurium-infected gnotobiotic mice. PLOS One 2013, 8(9):e74963.

50. Salzman NH, Hung K, Haribhai D, Chu H, Karlsson-Sjoberg J, Amir E, Teggatz P, Barman M, Hayward $\mathrm{M}$, Eastwood $\mathrm{D}$ et at. Enteric defensins are essential regulators of intestinal microbial ecology. Nat Immuno/2010, 11(1):76-83.

51. den Besten G, van Eunen K, Groen AK, Venema K, Reijngoud DJ, Bakker BM: The role of short-chain fatty acids in the interplay between diet, gut microbiota, and host energy metabolism. J Lipid Res 2013, 54(9):2325-2340.

52. Martin-Nunez GM, Cornejo-Pareja I, Coin-Araguez L, Roca-Rodriguez MDM, Munoz-Garach A, Clemente-Postigo M, Cardona F, Moreno-Indias I, Tinahones FJ: H. pylori eradication with antibiotic treatment causes changes in glucose homeostasis related to modifications in the gut microbiota. PLoS One 2019, 14(3):e0213548.

53. Rautio M, Eerola E, Vaisanen-Tunkelrott ML, Molitoris D, Lawson P, Collins MD, JousimiesSomer H: Reclassification of Bacteroides putredinis (Weinberg et al., 1937) in a new genus Alistipes gen. nov., as Alistipes putredinis comb. nov., and description of Alistipes finegoldii sp. nov., from human sources. Syst App/ Microbio/2003, 26(2):182-188. 
54. Kim JH, Doo EH, Jeong M, Kim S, Lee YY, Yang J, Lee JS, Kim JH, Lee KW, Huh CS et al: Enhancing immunomodulatory function of red Ginseng through fermentation using Bifidobacterium animalis Subsp. lactis LT 19-2. Nutrients 2019, 11(7).

55. Lukovac S, Belzer C, Pellis L, Keijser BJ, de Vos WM, Montijn RC, Roeselers G: Differential modulation by Akkermansia muciniphila and Faecalibacterium prausnitzii of host peripheral lipid metabolism and histone acetylation in mouse gut organoids. mBio 2014, 5(4).

56. Chambers ES, Viardot A, Psichas A, Morrison DJ, Murphy KG, Zac-Varghese SE, MacDougall K, Preston T, Tedford C, Finlayson GS et al: Effects of targeted delivery of propionate to the human colon on appetite regulation, body weight maintenance and adiposity in overweight adults. Gut 2015, 64(11):1744-1754.

57. Willemsen LE, Koetsier MA, van Deventer SJ, van Tol EA: Short chain fatty acids stimulate epithelial mucin 2 expression through differential effects on prostaglandin $E(1)$ and $E(2)$ production by intestinal myofibroblasts. Gut 2003, 52(10):1442-1447.

58. Duscha A, Gisevius B, Hirschberg S, Yissachar N, Stangl GI, Eilers E, Bader V, Haase S, Kaisler J, David $C$ et at. Propionic acid shapes the multiple sclerosis disease course by an immunomodulatory mechanism. Cell 2020, 180(6):1067-1080 e1016.

59. Beyaz S, Mana MD, Roper J, Kedrin D, Saadatpour A, Hong SJ, Bauer-Rowe KE, Xifaras ME, Akkad A, Arias $E$ et al: High-fat diet enhances stemness and tumorigenicity of intestinal progenitors. Nature 2016, 531(7592):53-58.

60. Kim Y, Hwang SW, Kim S, Lee YS, Kim TY, Lee SH, Kim SJ, Yoo HJ, Kim EN, Kweon MN: Dietary cellulose prevents gut inflammation by modulating lipid metabolism and gut microbiota. Gut Microbes 2020:1-18. 


\section{Figure Legends}

Figure 1. Mice treated with $A$. muciniphila had enhanced epithelial development and showed a thicker layer of mucus in the SI.

(A) PAS staining of SI and quantification of goblet cell numbers and crypt heights. (B) SI of mice treated with A. muciniphila were fixed by water-free methanol-Carnoy's fixative and stained with anti-Muc2 antibody. (C) SI mRNA levels of Muc2. (D) Confocal image of Lysozyme $^{+}$Paneth cells and quantification. (E) SI mRNA levels of Lyz1. (F) Confocal image of SI stained by anti-Ki67 antibody. (G) Percentages of Lgr5 $\mathrm{GFP}^{+}$cells either expressing Ki67 or not in SI crypt. (H) mRNA levels of Dll1, Mathl, and Spedf1. Scale bars: $100 \mu \mathrm{m}$ (A); $20 \mu \mathrm{m}$ (B); $50 \mu \mathrm{m}$ (D); $5 \mu \mathrm{m}$ (F). Statistical analyses were done by two-tailed paired ttest. ${ }^{*} P<0.05,{ }^{* *} P<0.01,{ }^{* * *} P<0.001$.

Figure 2. Mice treated with $A$. muciniphila showed enhanced $\mathrm{Lgr5}^{+}$stem cell populations in $\mathrm{SI}$ and $\mathrm{SI}$ organoids.

Confocal images of Lgr5 GFP cells (A) and quantification of Lgr5 GFP cells (B) in SI crypts. Lgr5 GFP expression (C) and surface areas (D) of SI organoids. (E) mRNA levels of Lgr5 in SI tissues and organoids. (F) RNA in situ hybridization for Olfm4 and quantification of Olfm $4^{+}$cells in SI. (G) Confocal image of SI organoid stained by anti-Muc2 and anti-Lyz mAbs. (H) mRNA levels of Muc2 and Lyzl in SI tissues. Scale bars: $10 \mu \mathrm{m}(\mathrm{A}) ; 50 \mu \mathrm{m}(\mathrm{C})$; $50 \mu \mathrm{m}$ (F); $50 \mu \mathrm{m}$ (Muc2); and $20 \mu \mathrm{m}$ (Lyz) (G). Statistical analyses were done by two-tailed paired t-test. ${ }^{*} P<0.05,{ }^{* *} P<0.01,{ }^{* * *} P<0.001$. 
Figure 3. Administration of $A$. muciniphila activated $\mathrm{Wnt} / \beta$-catenin pathway to enhance proliferation of ISCs.

(A) mRNA levels of Wnt3, Axin2, and Ctnnb1 in SI tissues. (B) In situ hybridization for Wnt3 and Axin2 in SI crypts. (C) Confocal images of Wnt3 in SI. (D) Representative confocal images of $\beta$-catenin in SI (left); numbers of nuclear translocation and mean fluorescence intensity (MFI) of $\beta$-catenin (right). (E) Co-culture with Lgr5 GFP ISCs and Paneth cells in presence of Wnt-C59. (F) Surface area of organoid was scored 6 and 12 days after plating. Scale bars: $10 \mu \mathrm{m}$ (B); $20 \mu \mathrm{m}$ (C and D); $20 \mu \mathrm{m}$ in Day 6 and $50 \mu \mathrm{m}$ in Day 12 (E). Statistical analyses were done by two-tailed paired t-test. ${ }^{*} P<0.05,{ }^{* *} P<0.01,{ }^{* * *} P<0.001$.

Figure 4. A. muciniphila-derived metabolites promote SI organogenesis.

(A) Expression levels of Lgr5 GFP and surface area of SI organoid in presence of cecal contents. (B) mRNA levels of $L g r 5, L y z 1, M u c 2$, Wnt3, Axin2, and Ctnnb1 in SI organoid treated with cecal contents. (C) Heat map of short chain fatty acid (left) and quantification of acetic, propionic, and butyric acids (right) derived from cecal contents. (D) Representative bright field image and surface area of SI-derived organoid treated with cecal contents in absence or presence of Gpr41/Gpr43 antagonists. Scale bars: $50 \mu \mathrm{m}$ (A); $100 \mu \mathrm{m}$ (D). Statistical analyses were performed by two-tailed paired t-test. ${ }^{*} P<0.05,{ }^{* *} P<0.01,{ }^{* * *} P<$ 0.001 .

Figure 5. Administration of $A$. muciniphila results in change of gut microbiota composition and community structure. 
(A) Taxonomic cladogram from LEfSe. Dot size is proportional to taxon abundance. (B) Linear discriminant analysis (LDA) scores of differentially abundant taxa in fecal microbiome. LDA score >2. (C) Shannon index in fecal microbiome. (D) Principal coordinate analysis (PCoA) of weighted UniFrac distances in fecal microbiome. (E) Correlation network between SCFAs and fecal microbiome. Dot color represents phylum level. Dot size is proportional to taxon abundance. Statistical analyses were performed by two-tailed paired ttest. ${ }^{* *} P<0.01$.

Figure 6. Administration of $A$. muciniphila ATCC BAA-835 prevents mouse gut injury. (A) Timeline of gut injury experiment. (B) Pathology of SI by H\&E staining. (C) Representative confocal image and number of $\mathrm{Lgr}_{5} \mathrm{GFP}^{+}$cells in SI following treatment with radiation (R) and methotrexate (M; MTX). (D) Body weight changes of mice after treatment with $\mathrm{R}$ and MTX $(\mathrm{n}=3)$. (E) Representative bright field image and surface area of SI organoid mice treated with R and MTX. (F) Organoid-forming capacity of SI crypt obtained from mice after treatment with R and MTX. Scale bars: $100 \mu \mathrm{m}(\mathrm{B}) ; 20 \mu \mathrm{m}(\mathrm{C}) ; 50 \mu \mathrm{m}(\mathrm{E})$. Statistical analyses were performed by two-tailed paired t-test. ${ }^{*} P<0.05,{ }^{* * * *} P<0.001$.

Figure 7. Isolation of $A$. muciniphila strains and validation of ISC stemness promoted by $A$. muciniphila.

(A and B) Representative bright field image and surface area of SI organoids. (A) Treated with culture supernatant from A. muciniphila ATCC BAA-835 or 1 of 11 A. muciniphila isolates. (B) Treated with bacterial culture supernatant in absence or presence of Gpr41/Gpr43 antagonists. (C) mRNA expression levels of pyruvate dehydrogenase E1 
component $\alpha$ subunit $(p d h)$ and $\mathrm{Na}^{+}$transporting methylmalonyl-CoA / oxaloacetate decarboxylase $\beta$ subunit (mmd) in A. muciniphila ATCC BAA-835 or AK32 strains. (D) Representative bright field image and surface area of SI-derived organoids from mice treated with A. muciniphila ATCC BAA-835 or AK32 strains. (E) Quantification of goblet cells and crypt height in mouse SI. (F) mRNA levels of Lgr5, Lyz1, Muc2, Wnt3, Axin2, and Ctnnb1 in mouse SI tissue. Scale bars: $50 \mu \mathrm{m}(\mathrm{A}, \mathrm{B}, \mathrm{D}) ; 10 \mu \mathrm{m}$ (E). Statistical analyses were performed by two-tailed paired t-test. ${ }^{*} P<0.05,{ }^{* *} P<0.01,{ }^{* * *} P<0.001$. 


\section{Figures}
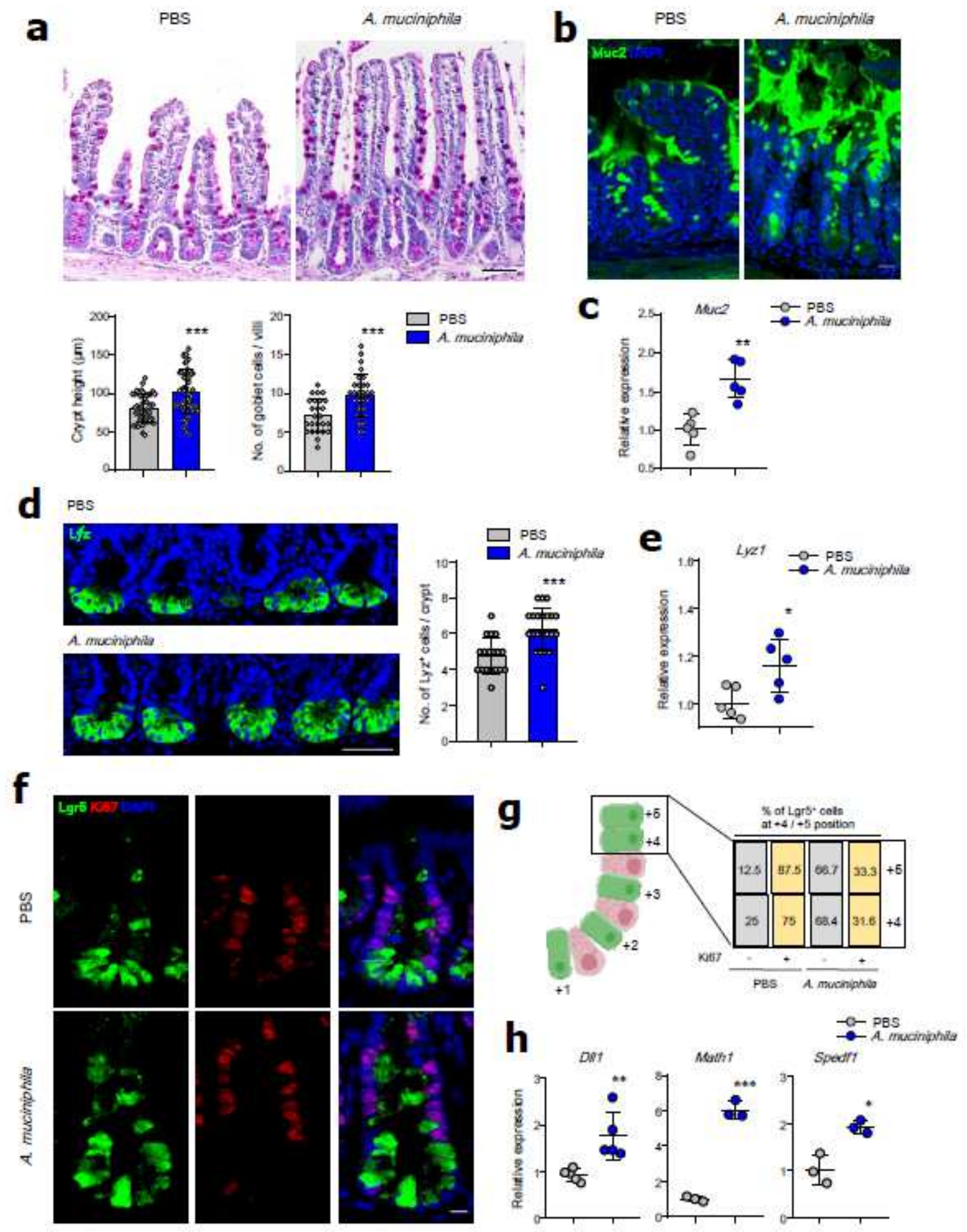

\section{Figure 1}

Mice treated with A. muciniphila had enhanced epithelial development and showed a thicker layer of mucus in the SI. (A) PAS staining of SI and quantification of goblet cell numbers and crypt heights. (B) SI of mice treated with A. muciniphila were fixed by water-free methanol-Carnoy's fixative and stained with 
anti-Muc2 antibody. (C) SI mRNA levels of Muc2. (D) Confocal image of Lysozyme+ Paneth cells and quantification. (E) SI mRNA levels of Lyz1. (F) Confocal image of SI stained by anti-Ki67 antibody. (G) Percentages of Lgr5 GFP+ cells either expressing Ki67 or not in SI crypt. (H) mRNA levels of DIl1, Math1, and Spedf1. Scale bars: $100 \mu \mathrm{m}(A) ; 20 \mu \mathrm{m}(\mathrm{B}) ; 50 \mu \mathrm{m}(\mathrm{D}) ; 5 \mu \mathrm{m}$ (F). Statistical analyses were done by two-tailed paired t- test. ${ }^{*} \mathrm{P}<0.05, * \star \mathrm{P}<0.01, * \star * P<0.001$.
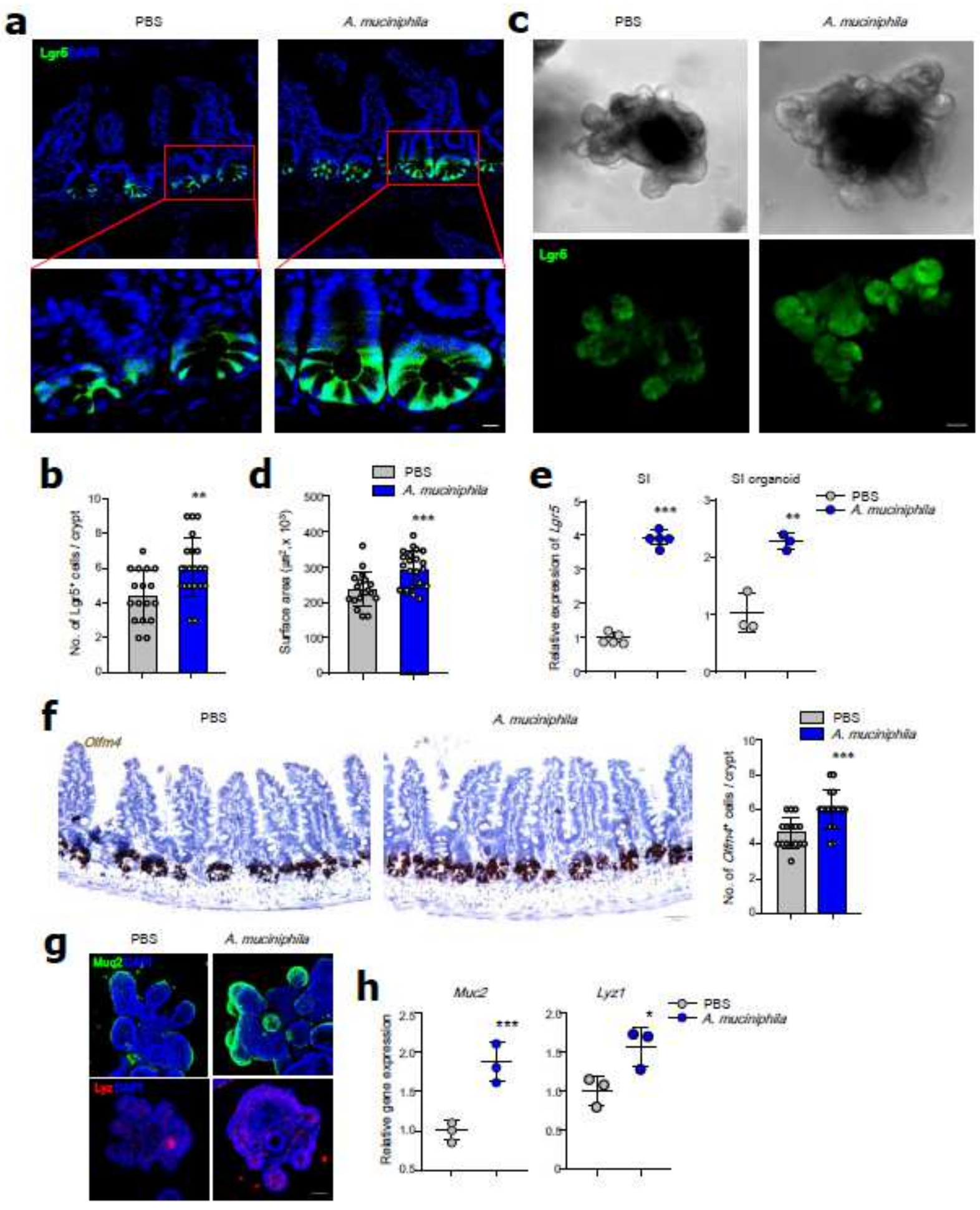

Figure 2 
Mice treated with A. muciniphila showed enhanced Lgr5+ stem cell populations in SI and SI organoids. Confocal images of Lgr5 GFP cells (A) and quantification of Lgr5 GFP cells (B) in SI crypts. Lgr5 GFP expression (C) and surface areas (D) of SI organoids. (E) mRNA levels of Lgr5 in SI tissues and organoids. (F) RNA in situ hybridization for Olfm4 and quantification of Olfm4+ cells in SI. (G) Confocal image of SI organoid stained by anti-Muc2 and anti-Lyz mAbs. (H) mRNA levels of Muc2 and Lyz1 in SI tissues. Scale bars: $10 \mu \mathrm{m}(\mathrm{A}) ; 50 \mu \mathrm{m}$ (C); $50 \mu \mathrm{m}$ (F); $50 \mu \mathrm{m}$ (Muc2); and $20 \mu \mathrm{m}$ (Lyz) (G). Statistical analyses were done by two-tailed paired t-test. * $P<0.05$, ** $P<0.01$, *** $P<0.001$.
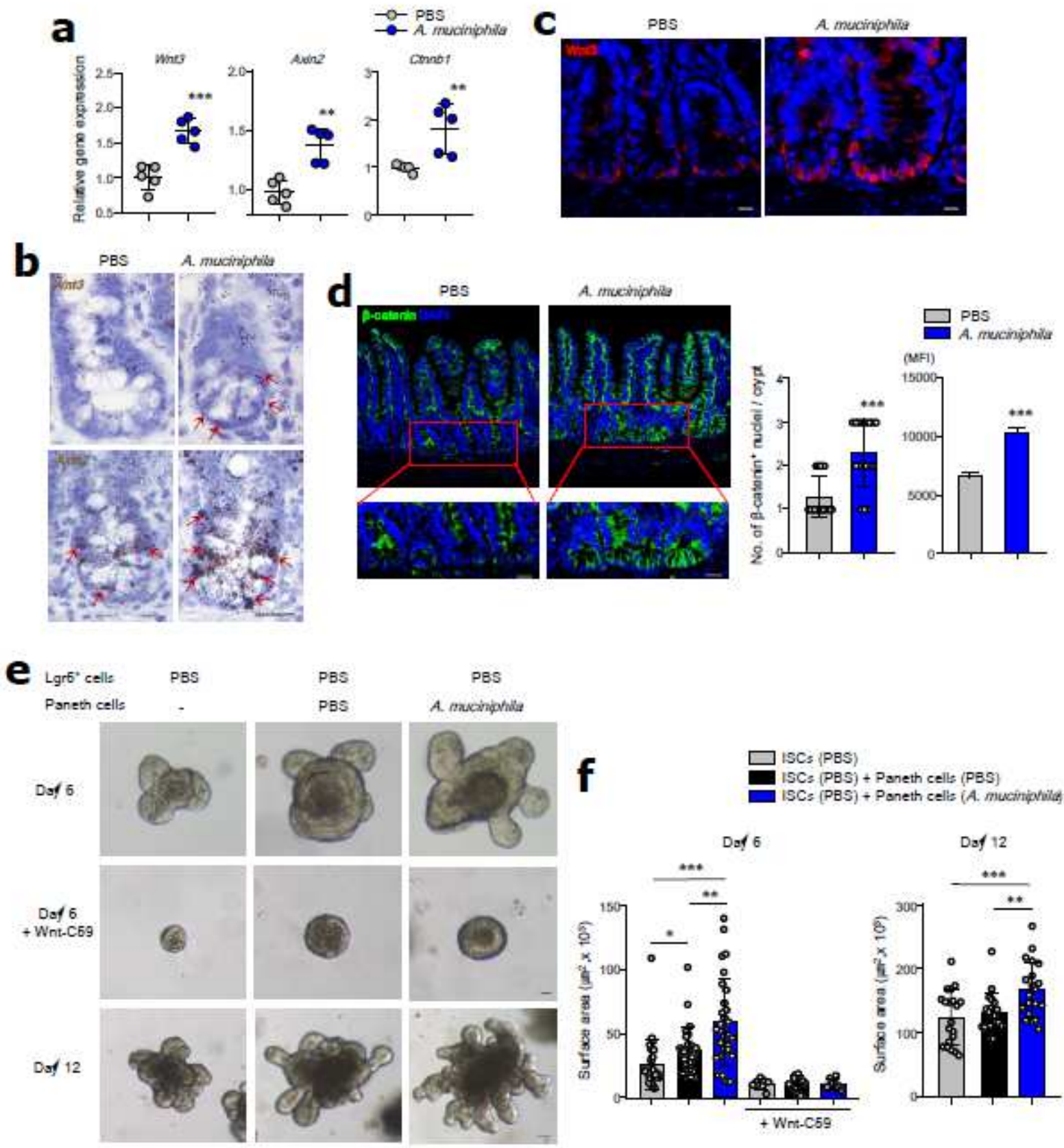

\section{Figure 3}

Administration of A. muciniphila activated Wnt/ $\beta$-catenin pathway to enhance proliferation of ISCs. (A) mRNA levels of Wnt3, Axin2, and Ctnnb1 in SI tissues. (B) In situ hybridization for Wnt3 and Axin2 in SI 
crypts. (C) Confocal images of Wnt3 in SI. (D) Representative confocal images of $\beta$-catenin in SI (left); numbers of nuclear translocation and mean fluorescence intensity (MFI) of $\beta$-catenin (right). (E) Coculture with Lgr5 GFP ISCs and Paneth cells in presence of Wnt-C59. (F) Surface area of organoid was scored 6 and 12 days after plating. Scale bars: $10 \mu \mathrm{m}$ (B); $20 \mu \mathrm{m}$ (C and D); $20 \mu \mathrm{m}$ in Day 6 and $50 \mu \mathrm{m}$ in Day 12 (E). Statistical analyses were done by two-tailed paired t-test. ${ }^{*} \mathrm{P}<0.05,{ }^{*} \mathrm{P}<0.01,{ }^{* *} \mathrm{P}<0.001$.
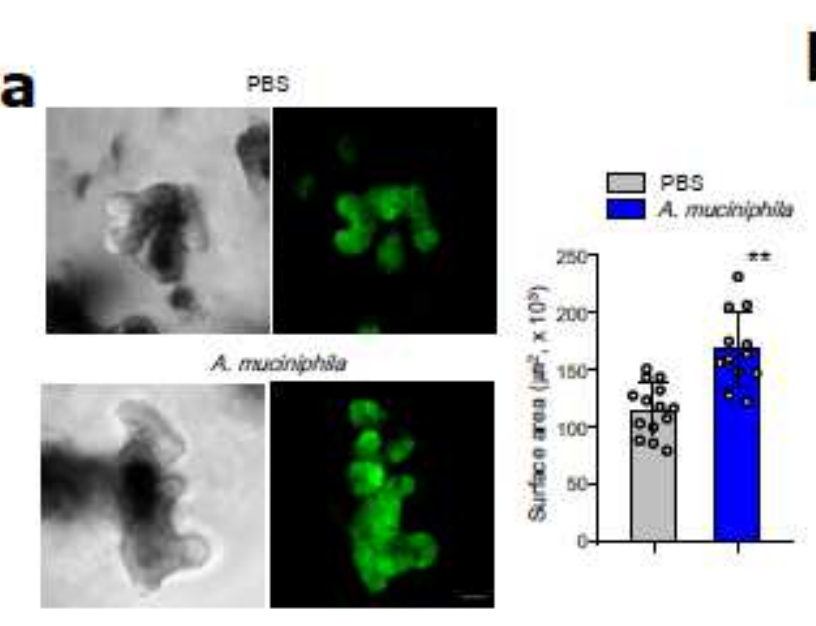

b

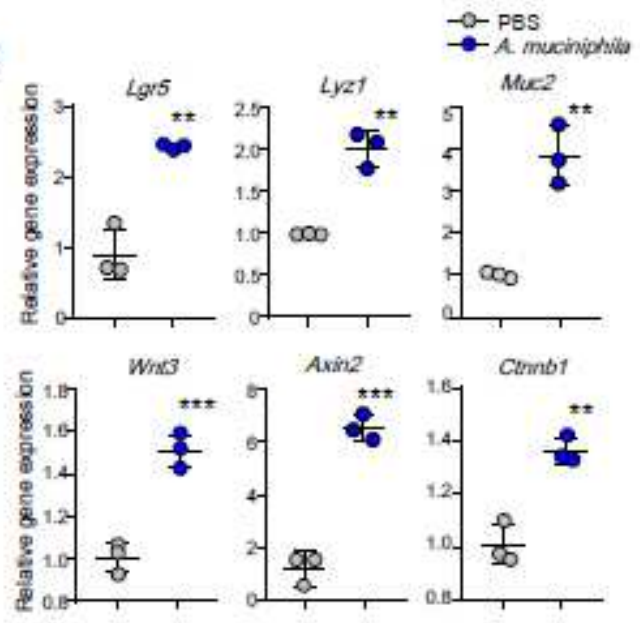

C
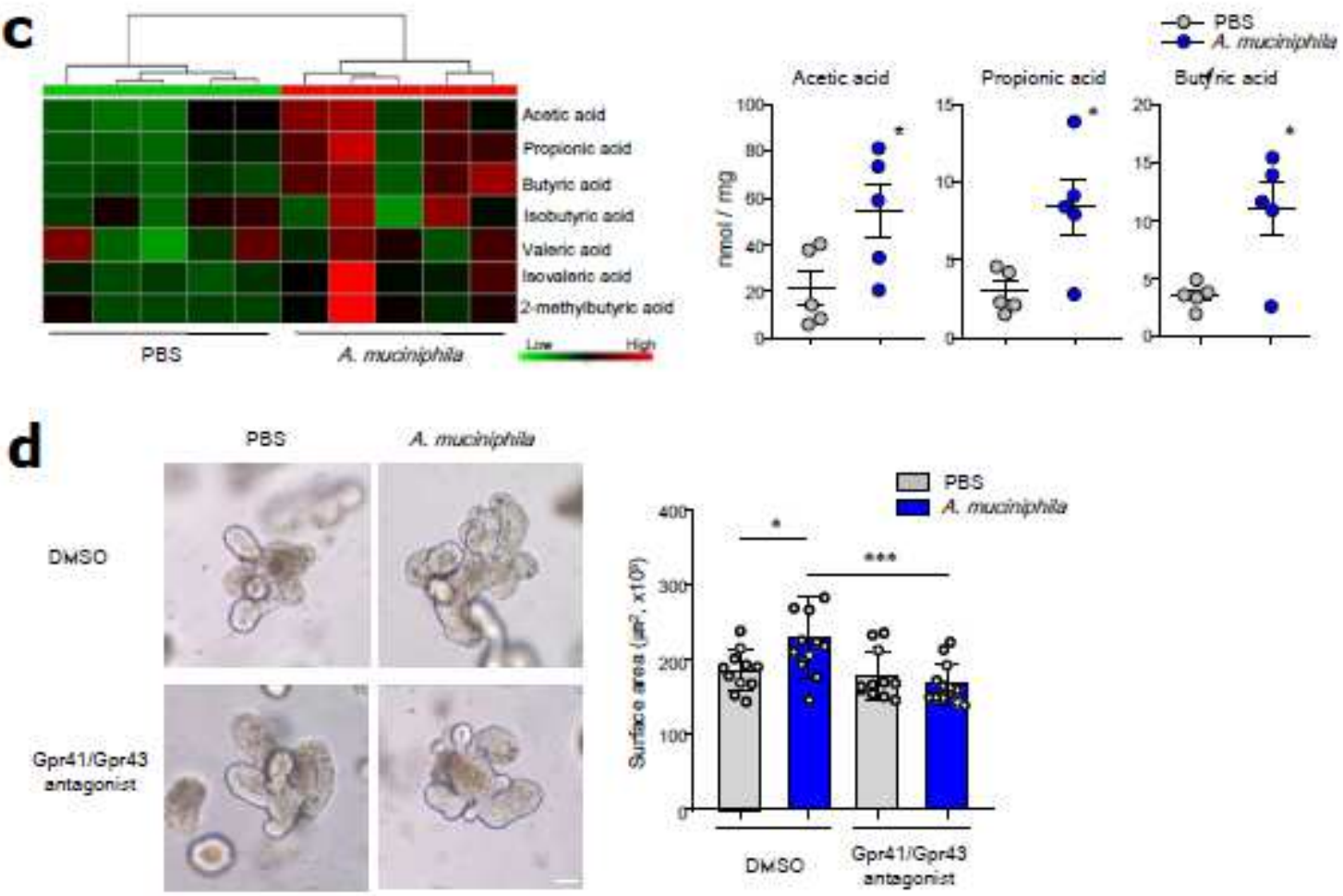

\section{Figure 4}

A. muciniphila -derived metabolites promote SI organogenesis. (A) Expression levels of Lgr5 GFP and surface area of SI organoid in presence of cecal contents. (B) mRNA levels of Lgr5, Lyz1, Muc2, Wnt3, Axin2, and Ctnnb1 in SI organoid treated with cecal contents. (C) Heat map of short chain fatty acid (left) and quantification of acetic, propionic, and butyric acids (right) derived from cecal contents. (D) 
Representative bright field image and surface area of SI-derived organoid treated with cecal contents in absence or presence of Gpr41/Gpr43 antagonists. Scale bars: $50 \mu \mathrm{m}(\mathrm{A}) ; 100 \mu \mathrm{m}$ (D). Statistical analyses were performed by two-tailed paired t-test. ${ }^{*} P<0.05$, ** $P<0.01$, *** $P<0.001$.
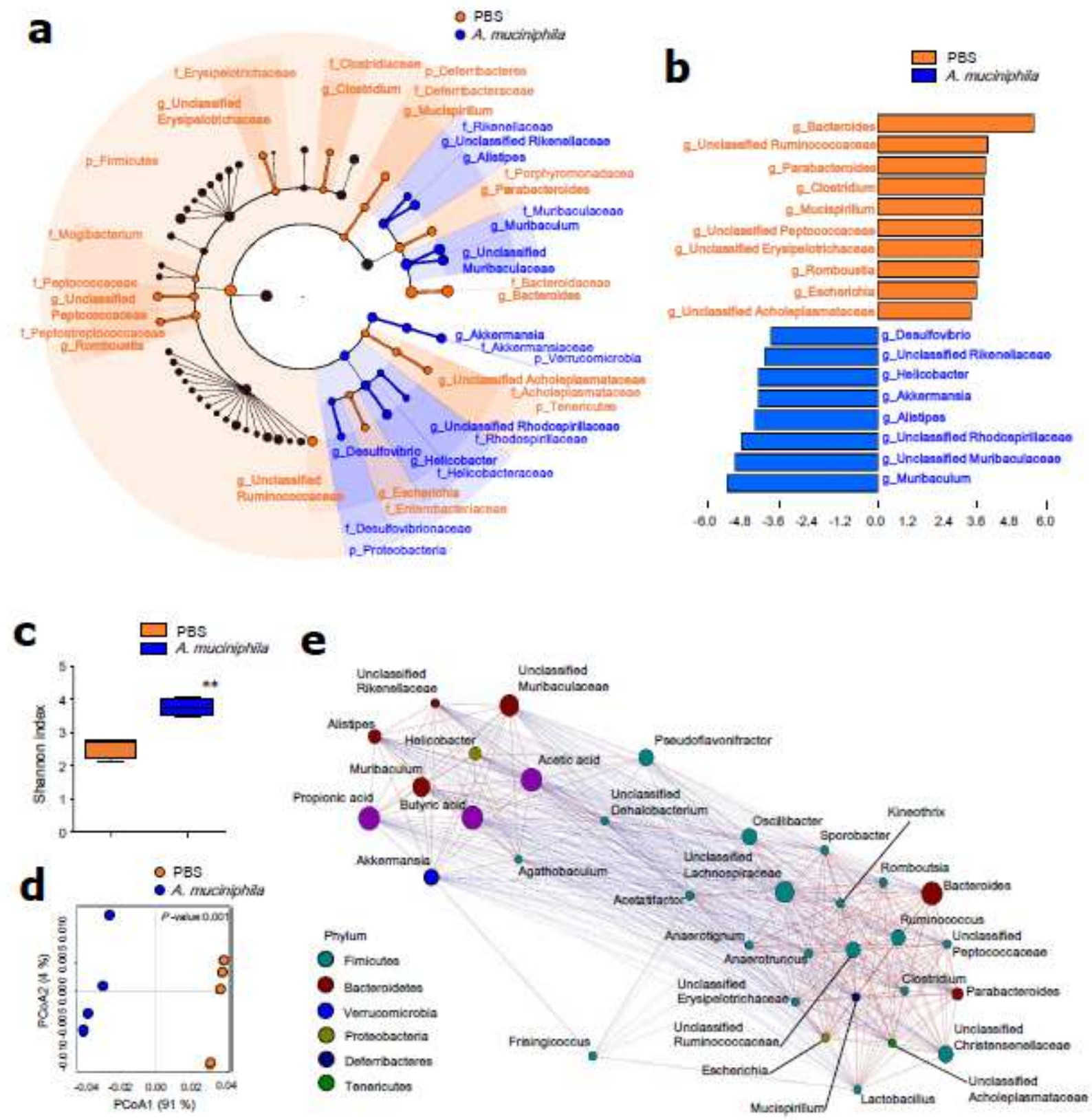

\section{Figure 5}

Administration of A. muciniphila results in change of gut microbiota composition and community structure. (A) Taxonomic cladogram from LEfSe. Dot size is proportional to taxon abundance. (B) Linear discriminant analysis (LDA) scores of differentially abundant taxa in fecal microbiome. LDA score $>2$. (C) Shannon index in fecal microbiome. (D) Principal coordinate analysis (PCoA) of weighted UniFrac distances in fecal microbiome. (E) Correlation network between SCFAs and fecal microbiome. Dot color 
represents phylum level. Dot size is proportional to taxon abundance. Statistical analyses were performed by two-tailed paired t- test. ** $\mathrm{P}<0.01$.


A. muciniphila $+\mathrm{R}+\mathrm{M}$

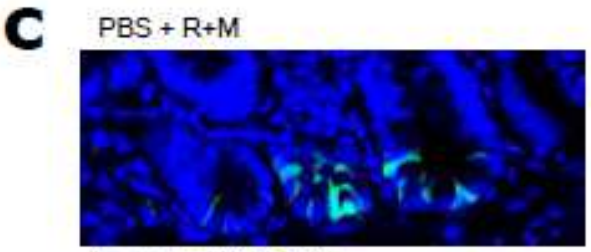

A. muciniphila $+\mathrm{R}+\mathrm{M}$
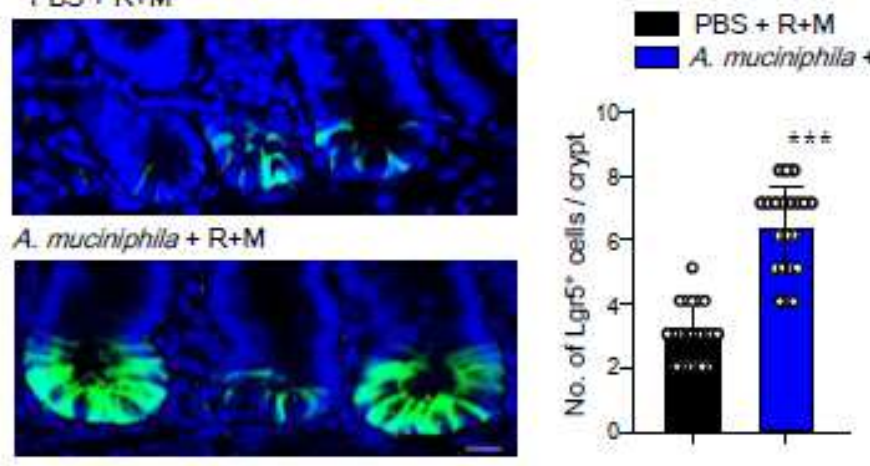

d
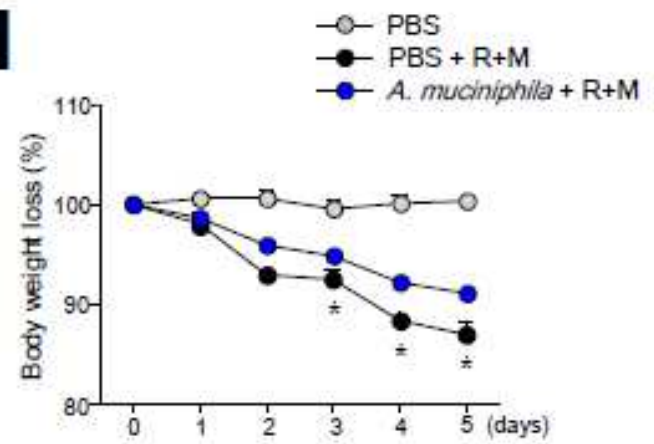


\section{Figure 6}

Administration of A. muciniphila ATCC BAA-835 prevents mouse gut injury. (A) Timeline of gut injury experiment. (B) Pathology of SI by H\&E staining. (C) Representative confocal image and number of Lgr5 GFP+ cells in SI following treatment with radiation (R) and methotrexate (M; MTX). (D) Body weight changes of mice after treatment with R and $\operatorname{MTX}(n=3)$. (E) Representative bright field image and surface area of SI organoid mice treated with R and MTX. (F) Organoid-forming capacity of SI crypt obtained from mice after treatment with R and MTX. Scale bars: $100 \mu \mathrm{m}(\mathrm{B}) ; 20 \mu \mathrm{m}(\mathrm{C}) ; 50 \mu \mathrm{m}(\mathrm{E})$. Statistical analyses were performed by two-tailed paired t-test. ${ }^{*} \mathrm{P}<0.05, * \star * \mathrm{P}<0.001$. 



- - BAA.935

d
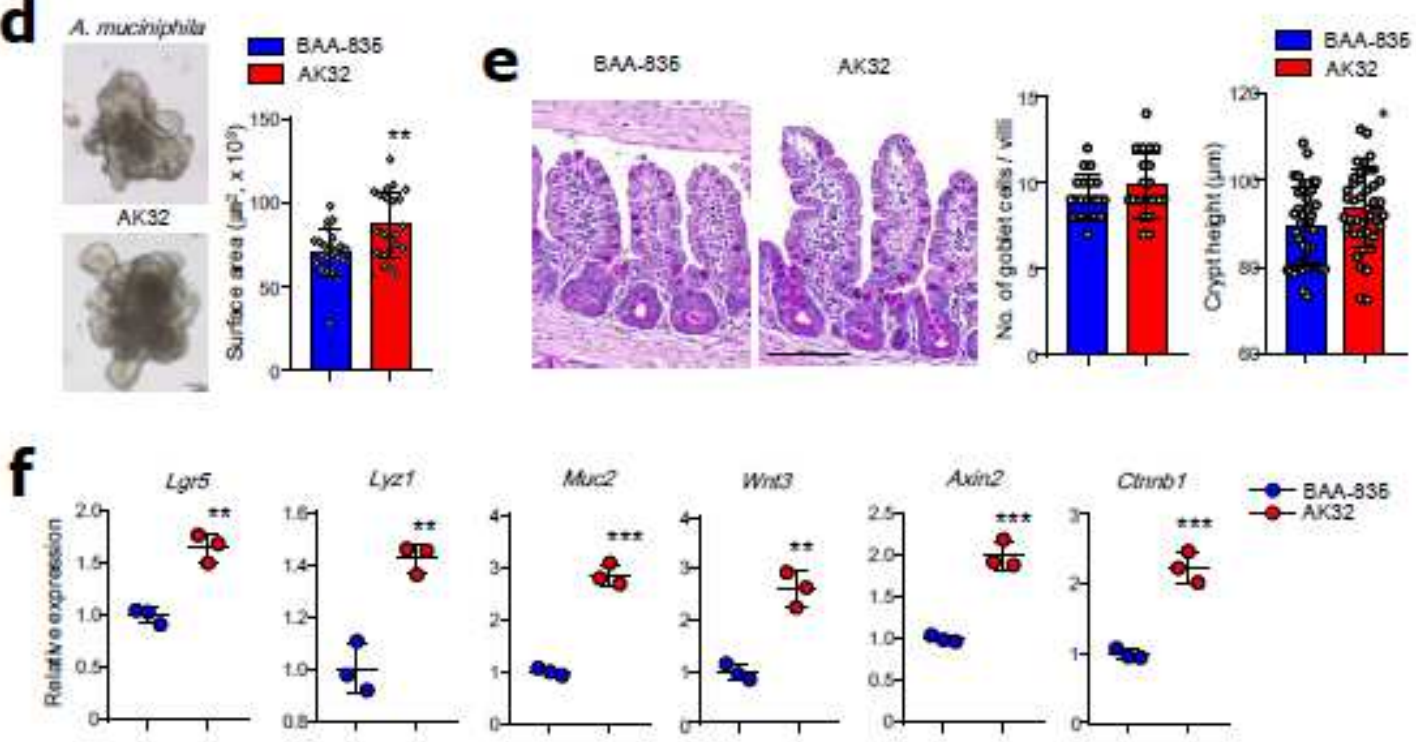

\section{Figure 7}

Isolation of A. muciniphila strains and validation of ISC stemness promoted by A. muciniphila. (A and B) Representative bright field image and surface area of SI organoids. (A) Treated with culture supernatant from A. muciniphila ATCC BAA-835 or 1 of 11 A. muciniphila isolates. (B) Treated with bacterial culture supernatant in absence or presence of Gpr41/Gpr43 antagonists. (C) mRNA expression levels of pyruvate dehydrogenase E1 component a subunit (pdh) and $\mathrm{Na}+$ transporting methylmalonyl-CoA / oxaloacetate decarboxylase $\beta$ subunit ( $\mathrm{mmd}$ ) in A. muciniphila ATCC BAA-835 or AK32 strains. (D) Representative 
bright field image and surface area of SI-derived organoids from mice treated with A. muciniphila ATCC BAA-835 or AK32 strains. (E) Quantification of goblet cells and crypt height in mouse SI. (F) mRNA levels of Lgr5, Lyz1, Muc2, Wnt3, Axin2, and Ctnnb1 in mouse SI tissue. Scale bars: $50 \mu \mathrm{m}(A, B, D) ; 10 \mu \mathrm{m}(E)$. Statistical analyses were performed by two-tailed paired t-test. $* P<0.05, * * P<0.01, * * * P<0.001$.

\section{Supplementary Files}

This is a list of supplementary files associated with this preprint. Click to download.

- AkkermansiaSupplementaryFiguresTableforMicrobiome.pdf 